\title{
Extended Reeb Graphs for Surface Understanding and Description
}

\author{
Silvia Biasotti, Bianca Falcidieno, and Michela Spagnuolo \\ Istituto per la Matematica Applicata, \\ Consiglio Nazionale delle Ricerche \\ \{silvia,falcidieno, spagnuolo\}@ima.ge.cnr.it \\ http://www.ima.ge.cnr.it/
}

\begin{abstract}
The aim of this paper is to describe a conceptual model for surface representation based on topological coding, which defines a sketch of a surface usable for classification or compression purposes. Theoretical approaches based on differential topology and geometry have been used for surface coding, for example Morse theory and Reeb graphs. To use these approaches in discrete geometry, it is necessary to adapt concepts developed for smooth manifolds to discrete surface models, as for example piecewise linear approximations. A typical problem is represented by degenerate critical points, that is non-isolated critical points such as plateaux and flat areas of the surface. Methods proposed in literature either do not consider the problem or propose local adjustments of the surface, which solve the theoretical problem but may lead to a wrong interpretation of the shape by introducing artefacts, which do not correspond to any shape feature. In this paper, an Extended Reeb Graph representation (ERG) is proposed, which can handle degenerate critical points, and an algorithm is presented for its construction.
\end{abstract}

\section{Introduction}

Reasoning about shape is a common way of describing real objects in engineering, architecture, medicine, biology, physics and in daily life. So far, research in modelling shapes has mainly focused on geometry, with the aim of defining effective representations and accurate approximations of objects [1]. To describe geometric objects, however, different levels of mental models can be used. It is possible to use natural-language terms to qualitatively describe the external shape of an object, or to draw a sketch of it, or to describe it by listing its differences with respect to some other similar objects, or also to define it according to what it is used for, and so on.

Shape recognition and classification are therefore basic steps for constructing descriptions of objects. These abstraction processes have been effectively described for example for biological taxonomy, where shape descriptions have been constructed by finding a good representation and then discarding irrelevant details $[2,3]$. The important point here is that reasoning about shape is an efficient approach not only for building high-level modelling environments, but 
also for devising top-down simplification methods based on the comprehension of the object shape [1].

Differential topology and geometry have been often used for surface coding, $[4,5,6]$, and building shape descriptions which nicely correspond to intuitive mechanisms of shape cognition and recognition.

For example an interesting method for coding the critical points of smooth Morse functions of two variables has been studied by Nackman [4]. Pfaltz proposed a similar approach for discrete surfaces defining the so-called surface networks [6]. Bajaj et al. [7] propose two different approaches, one bottom-up the other top-down, to preserve the topology during the simplification of discrete data. Their method, however, does not guarantee an exhaustive description of characteristics of the surface. Moreover, it does not allow to extract global properties of the surface and to detect degenerate configurations.

Major research on the use of topology for surface description has been proposed by Kunii, Shinagawa et al. in [5,8] where a surface coding based on Morse theory and Reeb graphs has been defined (see next section). Takahashi et al. [9], use an approach based on surface-networks to reconstruct the Reeb graph of a $2.5 D$ surface. As the previous references also this approach does not consider degenerate configurations which are typical of discrete surfaces, such as plateaux or flat areas.

In this paper, the Extended Reeb Graph (ERG) representation is proposed, which can handle degenerate critical points, and an algorithm is presented for constructing the ERG of bi-variate surfaces (scalar fields). The proposed extension does not distort the semantic meaning of the Reeb graph and faithfully represent the surface morphology.

The reminder of this paper is organised as follows: first, basic results of Morse and Reeb graph theory are given to introduce the proposed method; then our extension from the critical points concept to the critical areas is presented for discrete surfaces; finally, the proposed ERG representation and an algorithm for its construction from a set of contour levels are described. Some examples of its application to real surfaces are shown.

\section{Theoretical Background}

Morse theory originates from the calculus of variations and it allows describing differentiable manifolds using a limited number of information, for example by coding the topological relationships between critical points [10,11]. Using Morse theory, it is possible to construct topological spaces equivalent to a given smooth surface, which describe the surface as a decomposition into primitive topological cells [12]. Some basic results of Morse theory are given.

Definition 1. (Morse function) Let $f, f: M \rightarrow \mathbb{R}$, be a real smooth function defined on the smooth manifold $M ; f$ is called Morse function if all of its critical points are non-degenerate. A critical point is non-degenerate if the Hessian matrix $H$ of $f$ is non-singular at that point. 
Non-degenerate critical points are isolated, therefore scalar fields having plateaux or volcano rims do not comply with the definition of Morse function.

An example of a simple Morse function is given in figure 1(a). The figure 1(b) depicts an example of a complex Morse function, i.e. a function having more than one critical point at the same level, while figure 1(c) does not comply with the Morse's function definition.

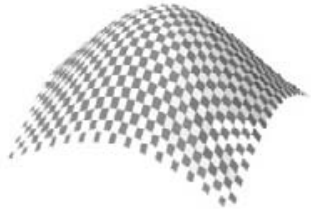

(a)

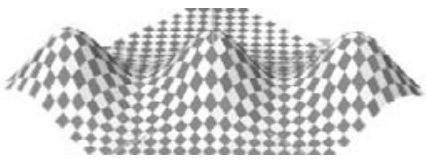

(b)

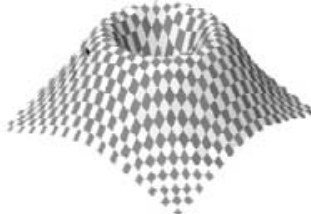

(c)

Fig. 1. An example of height function with an isolated maximum, (a), a non simple height function, (b), and a circle of non isolated maxima, (c). In (b) the saddle points as well as the maximum points have indeed the same elevation

The height function can be effectively used to study the surface shape. Intuitively, the height function of a smooth manifold $M$, embedded into the usual three-dimensional Euclidean space, is the real function which associates to each point on the surface its elevation.

Therefore, the level sets of a height function associated to a surface are the intersections of the surface with planes orthogonal to a fixed directions, which may be shaped in arbitrarily complex ways. If the height function is Morse, then the contour configuration can be quite simply classified. Moreover, smooth surfaces satisfy the Euler formula which states that the number of nondegenerate maximum $(M)$, saddle $(p)$ and minimum $(m)$ points satisfies the relation $M-p+m=2(1-g)$ where $g$ represents the genus of the surface.

Reeb defined a graph to code the evolution and the arrangement of level curves [5,13]. The Reeb graph of a function is defined as follows:

Definition 2. (Reeb graph) Let $f: M \rightarrow \mathbb{R}$ be a real valued function on a compact manifold $M$. The Reeb graph of $M$ wrt $f$ is the quotient space of $M x \mathbb{R}$ defined by the equivalence relation " ", given by

$$
(X 1, f(X 1)) \sim(X 2, f(X 2)) \Leftrightarrow f(X 1)=f(X 2)
$$

and $X 1$ and $X 2$ are in the same connected component of $f^{-1}(f(X 1))$

A Reeb graph of a compact manifold collapses into one element all points having the same value under a real function and being in the same connected component. Moreover, theorem 1 allows us to define a further equivalence relation among elements of the Reeb's quotient space. 
Theorem 1. Let $f$ be a real smooth function on a compact smooth surface $M$, and assume that the segment $[a, b]$ (where $a<b$ ) contains no critical value of $f$. Then:

- the level set $f^{-1}(a)$ and $f^{-1}(b)$ are diffeomorfic and they consist of the same number of smooth circles diffeomorfic to the standard circle;

- let $M_{a}$ be the subset of $M$ defined $M_{a}=\{x: f(x) \leq a\}$, and $M_{b}$ defined accordingly. Then $M_{a}$ and $M_{b}$ are diffeomorfic as two-manifolds with boundary.

In figure 2(a) the points drawn on the manifold represent the equivalence classes: here the manifold considered is a bi-torus and the function defined over it is the height function. In figure 2(b) the Reeb's quotient space is represented as a "traditional" graph where the equivalence classes are grouped into arcs if they are representative of diffeomorfic contours, as stated in the theorem 1 . Obviously, since the choice of the mapping function is not unique, a manifold admits different Reeb graphs.

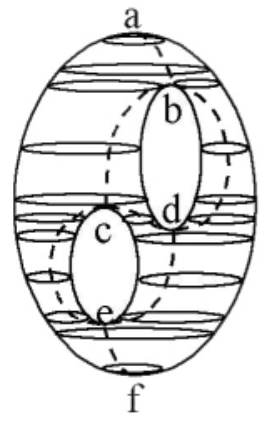

(a)

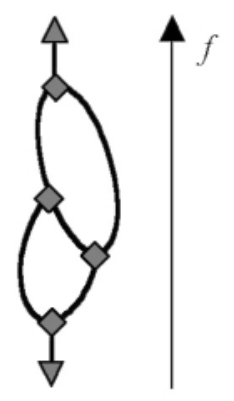

(b)

Fig. 2. A bi-torus, its contour lines and the equivalence classes defined by Reeb's quotient space (a), a possible representation of the Reeb graph wrt the function $f$, (b)

A Reeb graph describes a manifold surface by considering the evolution of the surface sections under a given real valued function. If we have to describe the shape of a manifold surface $M$ embedded into the Euclidean space, moreover, the Reeb graph of $M$ under its "natural" height function codes the shape description of $M$ in terms of critical points of $h$, corresponding to meaningful semantic labels, such as peaks, pits or passes. Moreover, under the assumption that the height function is Morse, the structure of the Reeb graph is rather simple. A Reeb graph of $M$ under $h$ can be defined as $R G_{h}(M)=\left(P_{h}(M), A_{h}(M)\right)$ where the node set is defined by $P_{h}(M)=\left\{P_{i} \in M, P_{i}\right.$ is a critical point of $\left.h(M)\right\}$ and the arc set is given by $A_{h}(M)=\left\{\left(P_{i}, P_{j}\right), P_{i}\right.$ and $P_{j} \in P_{h}(M)$ and the topological type of $M$ does not change between $P_{i}$ and $\left.P_{j}\right\} \quad$ (see Theorem 1). 
If $h(M)$ is Morse, moreover, the $\operatorname{arcs}$ of $R G_{h}(M)$ can be oriented and the nodes have almost degree three.

\section{Extended Reeb Graph Representation}

Reeb graph theory provides a powerful abstraction mechanism to describe the shape of smooth surfaces. Even if there are no restrictions in the Reeb graph definition on the type of $f$, several authors have in practice limited the use of the Reeb graph to Morse mapping functions (see section 1). When dealing with discrete surface representations, such as triangular meshes, problems may arise due to the loss of properties of the resulting surface which can be assumed to be at most continuous. The straightforward application of the Reeb graph definition to a generic polyhedral surface (e.g. a 3D triangulated manifold) requires at first the definition and extraction of the critical points. The possible definitions of discrete critical points, however, generally suffer of instability since small perturbations of the vertex coordinates may result in rather different configurations $[9,7]$.

This problem is particularly crucial for surfaces defined by measurements of some natural or physical objects, as in digital terrain modelling or reverse engineering, where the position of the mesh vertices may have some uncertainty associated. Therefore, we have adopted a different approach based on the computation of a "sufficiently" dense number of contour lines and the definition of the Reeb graph from the contour set. The evolution of contour lines is less sensible to local perturbation of vertex position and, globally, provides a faithful description of the surface shape. The same approach has been adopted by $\mathrm{Ku}-$ nii, Shinagawa et al., [5,8], for smooth surfaces for which the height function is Morse, while here generic continuous surfaces are considered for which a set of contour levels is given (or computed).

An extension of the Reeb graph representation $(E R G)$ is introduced, which can handle degenerate and non simple critical points, and an algorithm is presented for constructing the ERG automatically from the set of contour levels of a discrete surface. The proposed extension does not distort the semantic meaning of the Reeb graph and faithfully represents the surface morphology.

The innovation of this method is both the way of constructing the graph and the efficiency in dealing with degenerate situations. The proposed approach is actually not an extension of the Reeb graph itself, but rather a full application of its definition in the discrete domain, which does not require the height function to be Morse. The quotient space defined by the Reeb equivalence relation can be represented in terms of a graph representation, which formally represents the contour containment and the adjacency relationships. In this way, with an extended definition of critical areas, the application domain can be enlarged to generic continuous scalar fields, without any artifacts [14].

\subsection{Definition of Critical Areas and Influence Zones}

First of all, to get a more general and unambiguous shape description, we extend the idea of critical points to critical areas as well. With respect to other 
definitions, the idea of critical areas provides a more general approach as it takes into consideration the behaviour of a surface in a neighbourhood rather than locally around a point.

Formally, let $M$ be a continuous surface defined by a scalar field $f: D \subset$ $\mathbb{R}^{2} \rightarrow \mathbb{R}$, that is, $M=\{P=(x, y, z) \mid z=f(x, y)\}$ and let $h$ be the height function naturally defined over $M, h(P): M \rightarrow \mathbb{R}$ such that $h(P)=h((x, y, f(x, y)))$ $=f(x, y)$ and let $P_{i}$ be the critical points of $h(M)$. Under the assumption that $M$ is Morse, $f^{-1}\left(P_{i}\right)$ is an isolated critical point of the surface, while if we do not require $M$ is Morse, $f^{-1}\left(P_{i}\right)$ generally represents a critical area of the surface, a level region of $M$. Let $C(M)$ be the set of contour levels of $M$; we assume that $C(M)$ is a set of unorganised polygonal contours, which are either simple closed polygons or polygonal lines with the end points on the boundary of the surface.

Let $C_{T}(M)$ be the Delaunay triangulation of $C(M)$ constrained to all contours lines. The critical points of $h(M)$ can be efficiently detected by classifying the flat regions of $C_{T}(M)$, that is, the connected regions of $C_{T}(M)$ composed by triangles having all the three vertices at the same elevation $[15,16,17]$.

The flat regions of $C_{T}(M)$ correspond either to simply or to multiply-connected areas. Let $B_{R}(M)$ be the boundary of a critical area $R$ of $C_{T}(M)$, in general $B_{R}(M)=B_{1} \cup B_{2} \cup \ldots \cup B_{n}$ where $B_{i}$ represents the $i-t h$ connected component of the boundary. According to the definition of critical area, each $B_{i}$ may be either a contour in $C(M)$, having elevation $h\left(B_{i}\right)$ or may be a sequence of edges of $C_{T}(M)$, as for saddle areas. Simply-connected critical areas, for which $B_{R}(M)=B_{1}$, correspond either to isolated or degenerate critical points of $M$. A multiply- connected critical area divides the surface into two parts: an "outer" part which is defined by the portion of the surface outside the boundary of the multiply-connected area, and as many "inner" parts as the multiplicity of the critical area boundary. Let $B_{1}$ be the outer boundary component and $B_{i}$, with $i>1$, the inner ones. The following classification scheme is adopted:

- $B_{R}(M)$ is a simple maximum area (resp. simple minimum area) iff $n=$ 1 and the outgoing direction from $B_{1}$ is descendent (resp. ascending), see figure $3(\mathrm{a})$;

- $B_{R}(M)$ is a complex maximum area (resp. complex minimum area) iff $n>1$ and the outgoing directions from each $B_{i}$ are descendent (resp. ascending), (see figure 3(b));

- $B_{R}(M)$ is a simple saddle area iff $n=1$ and there are at least four outgoing directions alternatively descendent and ascendent;

$-B_{R}(M)$ is a complex saddle area iff $n>2$ and the outgoing directions are descendent for some $B_{i}$ and ascending for some other $B_{j}$ or the outgoing direction for $B_{1}$ are either descendent and ascending.

When considering surfaces defined by scalar fields or more generally manifold surfaces with boundary it is necessary to give a unique interpretation to critical points occurring along the surface boundary by considering a global virtual minimum point as a virtual closing of the surface with descending directions from the boundary to a global minimum. 
Obviously, the flat regions of $C_{T}(M)$ do not always correspond to critical points of the height function: for example, ridges and ravines of $M$ will cause level regions to appear as well, with a step-like effect which is a very well-known problem in digital terrain modelling. These areas, however, can be easily detected considering the number of the ascending or descending directions of the region boundary, as explained in details in [14,17]. Therefore, let us assume from now that the critical areas only correspond to critical points of $h(M)$, either isolated or degenerate. Generally for polyedral surfaces the Eulero formula given in section 2 is not verified. Nevertheless considering the above defined critical areas for $C_{T}(M)$ the Eulero formula is still satisfied if they correspond to nondegenerate critical points .

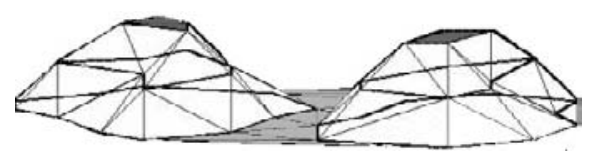

(a)

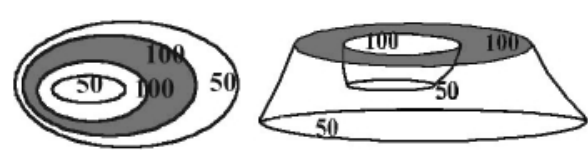

(b)

Fig. 3. Critical areas of $M$ correspond to flat regions in $C_{T}(M)$ : areas of maximum are depicted in dark and saddle areas in light grey

It is important to show the link between critical areas and Reeb graph nodes. By applying the definition of Reeb graph (cf. definition 2) all points belonging to a simply-connected critical area are Reeb-equivalent and may therefore collapse into the same node. If the isolated critical points of $M$ were known, moreover, a simple labelling of the graph's node set would be sufficient to distinguish them from degenerate critical points. Similarly, the behaviour of arcs incident to simple critical areas is equivalent to the behaviour of arcs incident to isolated critical points. Therefore, simple critical areas can be represented in the ERG by simple nodes as in the normal Reeb graph representation.

Multiply-connected critical areas correspond to macro-nodes: that is particular nodes having at least one arc connected to an inner node.

With respect to the Reeb graph arcs we have introduced the concepts of influence zones which are defined using adjacency among contours: two contours are adjacent if they are edge-adjacent in the constrained triangulation. To connect critical areas in the Reeb graph, an influence zone is associated to critical areas, which intuitively spans the surface between adjacent Reeb graph nodes.

First of all, if $R$ is a simple saddle critical area, its influence zone is defined as the surface region delimited by the contours which are directly edge-adjacent to $R$, in other words contours which are at a distance 1 from the saddle area.

Then, if $R$ is a maximum or minimum critical area, the influence zone of $R$ is the portion of the surface identified by growing $B_{1}$, the outmost component of the region boundary, until the boundary of another critical area is reached. Therefore, if the critical area is a simple maximum its influence area is the maximal region in $C_{T}(M)$ containing $R$ and non containing other critical areas. 
Similarly, the influence zone of a multiply-connected area does not contain any other critical areas except those located in its internal boundary components. In practice the influence zone definition for minimum and maximum areas strictly relates with the theorem 1: in fact the expansion process allows to connect level sets which are diffeomorfic. For saddle areas, indeed, the influence zone represents the portion of the surface where the topological change arises. In figure 4, some critical areas are shown with the corresponding influence zones. The dark colour identifies maximum (a) and saddle areas (c), their corresponding influence zones are depicted in medium grey colour, (b) and (d).

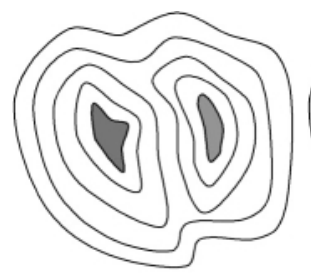

(a)

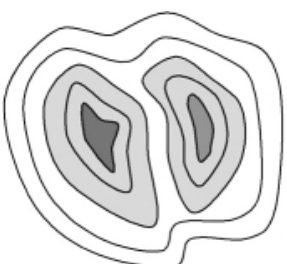

(b)

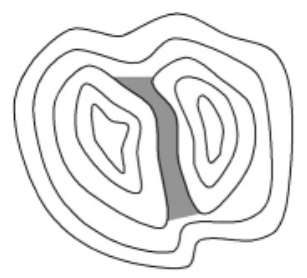

(c)

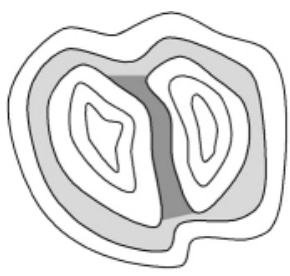

(d)

Fig. 4. Critical areas, (a), (c) and their influence zones (b), (d)

\subsection{Construction of the ERG Representation}

In this paragraph a short description of the algorithm to extract the critical areas is given. First of all it is important to notice that each contour $C_{T}(M)$ is edge-adjacent to the previous and the next one, in the height sequence of $C(M)$; therefore the ascending/descending directions between contours can be simply checked. Then, critical points of $h(M)$ can be efficiently detected by classifying the level regions of $C_{T}(M)$, that is, the connected regions of $C_{T}(M)$ composed by triangles having all the vertices at the same elevation $[15,16,17]$. The classification of flat areas as critical areas, simple or complex, is done by checking the number of non-constrained edges in the boundary:

- simple saddle areas are detected in $C_{T}(M)$ as flat areas with more than one edge in the boundary, which does not belong to the constraints, that is to the contours;

- maximum or minimum areas, either simple or complex, and complex saddles are detected in $C_{T}(M)$ as flat areas whose boundary is fully composed by constrained edges of $C_{T}(M)$.

The distinction among the different types of critical areas is done by analyzing the coordinates of the vertices which are edge-adjacent to the region boundary, that is, by checking the ascending/descending directions, as in section 3.1. 
According to the graph representation of the Reeb's quotient space, each node of the graph corresponds to a critical point, or area, and each arc corresponds to a connected component of the manifold between two critical levels of the height function. This suggests to follow a similar approach for automatically extracting the Reeb graph, based on tracking the evolution of contour lines. First, the critical areas are recognised, and the $E R G$ is initialized by creating the node corresponding to the virtual minimum, $V M$. The $V M$ is connected to the saddle (complex or simple) having the minimum elevation and external to each macro-node. If such a saddle does not exist, then the $V M$ is connected to the first complex maximum area, otherwise the $E R G$ is a trivial graph connecting the $V M$ to the only simple maximum existing. Then, using the notion of influence zone, the Reeb graph arcs are partially computed from the adjacency relationships between influence zones. In this manner the arcs connected to the terminal nodes of the ERG are identified. To complete the ERG construction the links between saddles and, in general, complex areas, have to be determined. Intuitively, arcs correspond to ascending paths between critical areas which are determined by expanding the associated influence zones, following free directions in the outmost boundary component (see figure 5). Free directions are those which do not correspond to an already identified arc.

In the following, the construction algorithm is described using a $\mathrm{C}$ pseudocode. At each step, if the critical region is multiply-connected, then a macro-node is defined, with as many arcs as the inner components of the critical region. Note that the definition of influence zone guarantees that the Reeb graph is correctly constructed.

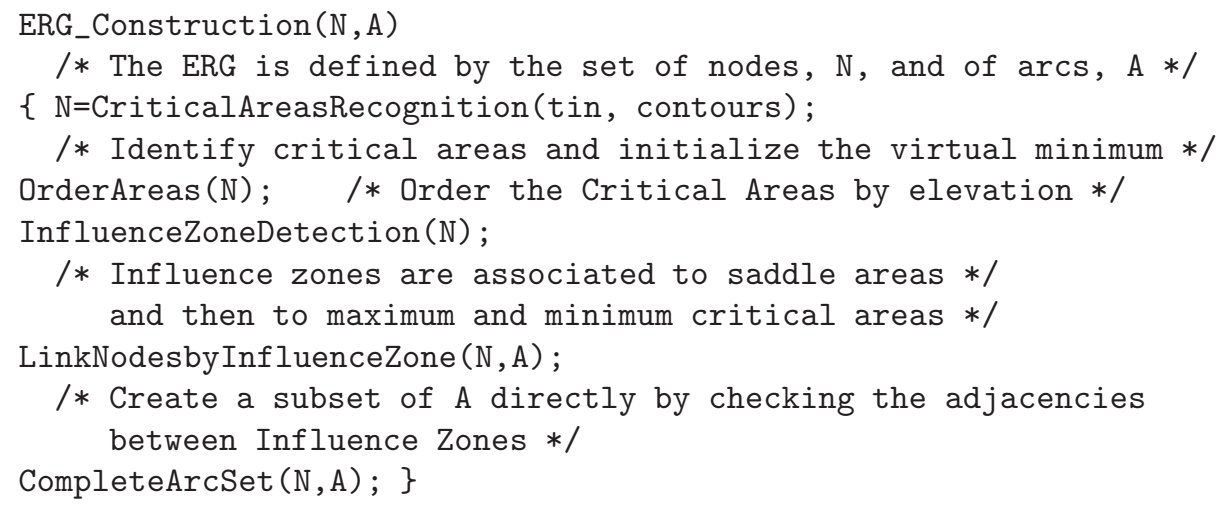

For the sake of clarity the function "CompleteArcSet" is here expanded:

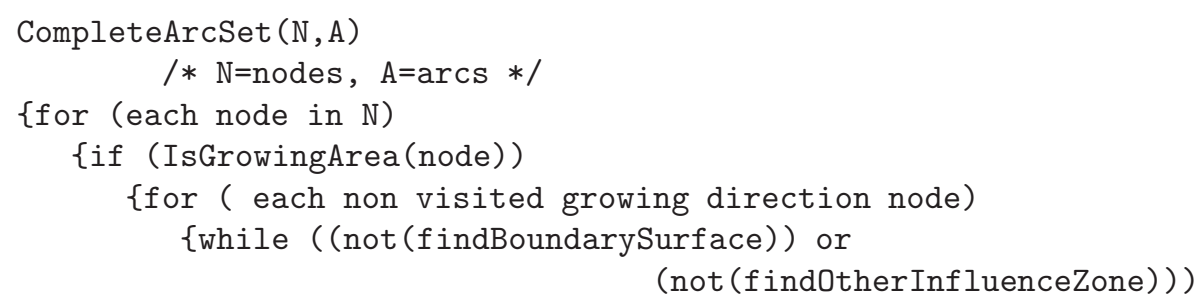




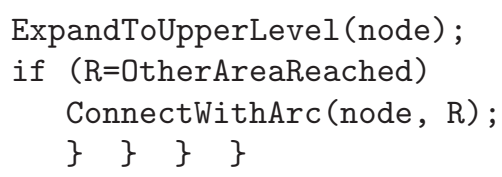

The function "IsGrowingArea(node)" returns a boolean value which is TRUE if the critical area has at least one growing direction which has not been visited yet.

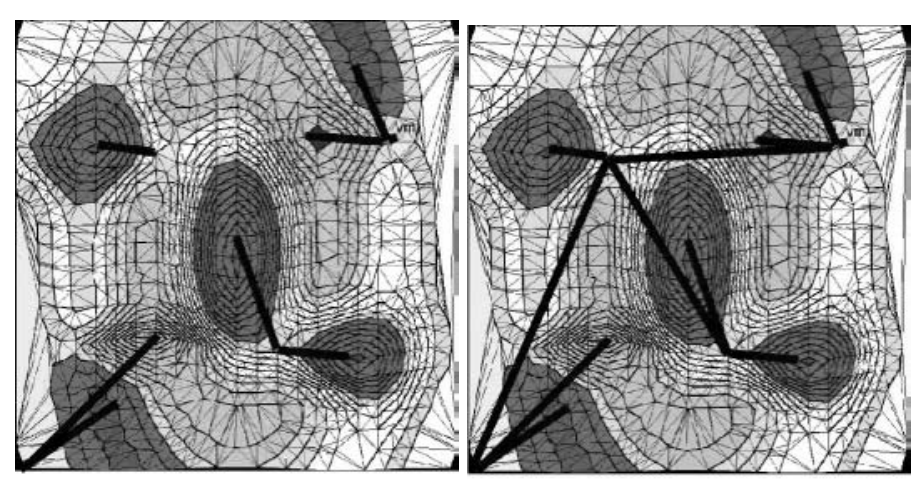

(a)

(b)

Fig. 5. Two steps of the Reeb graph reconstruction process

Some examples are given: in figure 6(a), a test surface is depicted with the critical areas identified on the triangulation; its Reeb graph is depicted in 6(b) and $6(\mathrm{c})$. The surface that can be trivially reconstructed by triangulating only the critical sections of the $E R G$ is shown in $6(\mathrm{~d})$ : even if rough, the reconstruction still faithfully reproduces the original surface morphology. Finally, in figure 7, another example of our characterisation method applied to a natural surface (a) is shown, then the reconstructed Reeb graph is depicted in (b) and (c) and the surface reconstructed considering only the critical sections is shown in (d).

\section{Conclusions and Future Developments}

In this paper a model has been defined which provides a high-level description of the surface shape. Morse theory concepts and Reeb graph properties have been used and translated to the discrete domain in order to define a sketch of the surface usable for classification or compression and decompression purposes [18].

With respect to previous work in this field, the proposed Extended Reeb Graph is able to faithfully represent the surface shape, even in case of degenerate critical points. The algorithm for constructing the ERG of scalar fields, represented as contour lines, is efficient and allows a topologically correct reconstruction of the surface shape. 


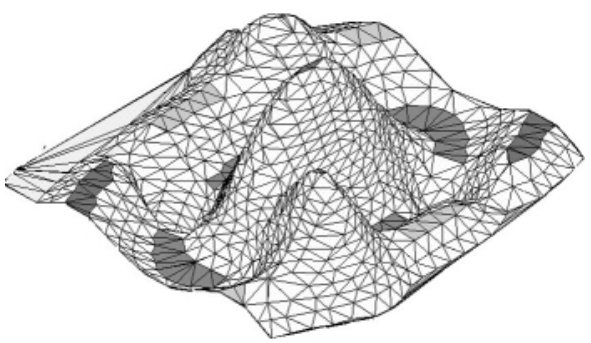

(a)

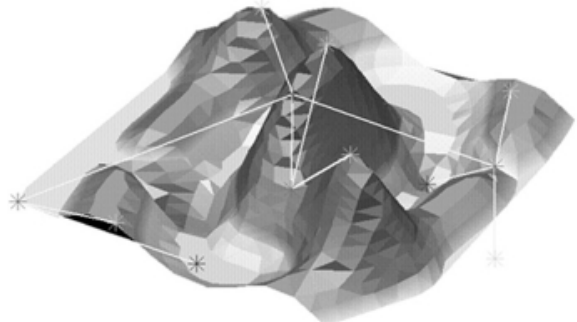

(c)

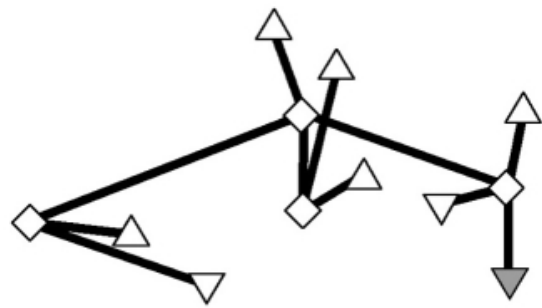

(b)

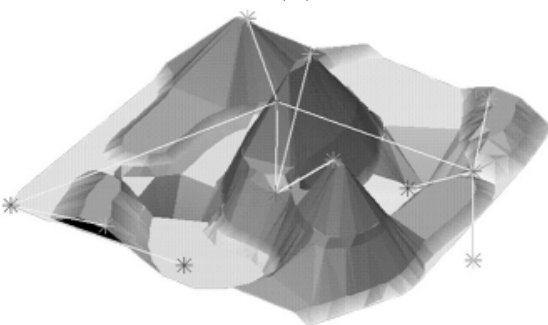

(d)

Fig. 6. A test surface (a), its Reeb graph (b) and (c) and the reconstructed model $(d)$

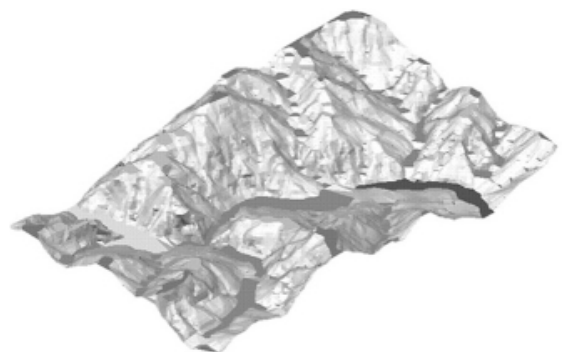

(a)

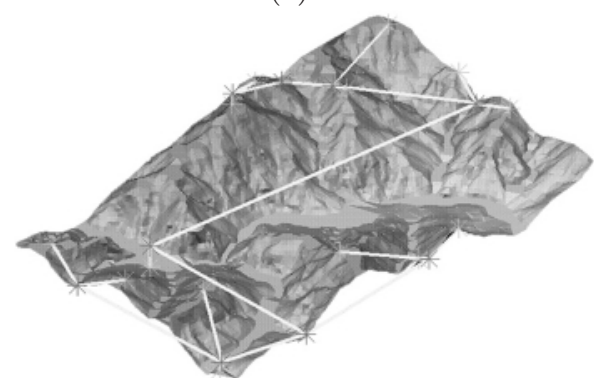

(c)

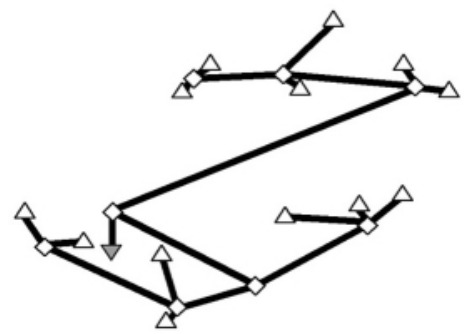

(b)

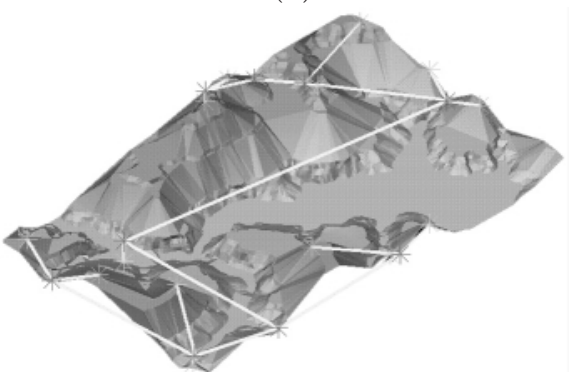

(d)

Fig. 7. The critical areas of a terrain (a), its Reeb graph (b), (c) and the recostruction from the critical sections (d) 
Future developments are currently under consideration, mainly for the extension of the method to three dimensional surfaces, with or without boundary. Moreover, the shape decompression process is also being developed, taking into account several shape reasoning steps which allow a better definition of shapebased generation of intermediate sections between critical sections, coded in the Reeb graph [18].

\section{Acknowledgements}

Thanks are given to Prof. Tosiyasu Kunii for the many helpful discussions on this topic, and to Corrado Pizzi for his helpful technical support.

\section{References}

1. B. Falcidieno, M. Spagnuolo. Shape Abstraction Paradigm for Modelling Geometry and Semantics. In Proceedings of Computer Graphics International, pp. 646-656, Hannover, June 1998. 185, 186

2. W. D'Arcy Thompson. On growth and form. MA: University Press, Cambridge, second edition, 1942185

3. P. Pentland. Perceptual organization and representation of natural form. Artificial Intelligence, Vol.28, pp. 293- 331, 1986. 185

4. L. R. Nackman. Two-dimensional Critical Point Configuration Graphs. IEEE Transactions on Pattern Analysis and Machine Intelligence, Vol. PAMI-6, No. 4, p. $442-450,1984.186$

5. Y. Shinagawa, T. L. Kunii, Y. L. Kergosien. Surface Coding Based on Morse Theory. IEEE Computer Graphics \& Applications, pp 66-78, September 1991. 186, 187, 189

6. J. L. Pfaltz. Surface Networks. Geographical Analysis, Vol. 8, pp. 77-93, 1990. 186

7. C. Bajaj, D. R. Schikore. Topology preserving data simplification with error bounds. Computer \& Graphics, 22(1), pp. 3-12, 1998. 186, 189

8. Y. Shinagawa, T. L. Kunii. Constructing a Reeb graph automatically from cross sections. IEEE Computer Graphics and Applications, 11(6), pp 44-51, 1991. 186, 189

9. S. Takahashi, T. Ikeda, Y.Shinagawa, T. L.Kunii, M. Ueda. Algorithms for Extracting Correct Critical Points and Construction Topological Graphs from Discrete geographical Elevation Data. Eurographics '95, Vol. 14, Number 3, 1995. 186, 189

10. A. Fomenko. Visual Geometry and Topology. Springer- Verlag, 1994. 186

11. V. Guillemin, A. Pollack. Differential Topology. Englewood Cliffs, NJ: PrenticeHall, 1974. 186

12. J. Milnor. Morse Theory. Princeton University Press, New Jersey, 1963. 186

13. G. Reeb. Sur les points singuliers d'une forme de Pfaff completement integrable ou d'une fonction numérique. Comptes Rendus Acad. Sciences, Paris, 222:847-849, 1946. 187

14. S. Biasotti. Rappresentazione di superfici naturali mediante grafi di Reeb. Thesis for the Laurea Degree, Department of Mathematics, University of Genova, September 1998. 189, 191 
15. G. Aumann, H. Ebner, L. Tang. Automatic derivation of skeleton lines from digitized contours. ISPRS Journal of Photogrammetry and Remote Sensing, 46, pp. 259-268, 1991. 190, 192

16. M. De Martino, M. Ferrino. An example of automated shape analysis to solve human perception problems in anthropology. International Journal of Shape Modeling, Vol. 2 No. 1, pp 69-84, 1996. 190, 192

17. C. Pizzi, M. Spagnuolo. Individuazione di elementi morfologici da curve di livello. Technical Report IMA, No. 1/98, 1998. 190, 191, 192

18. S. Biasotti, M. Mortara, M. Spagnuolo. Surface Compression and Reconstruction using Reeb graphs and Shape Analysis. Spring Conference on Computer Graphics, Bratislava, May 2000. 194, 196 